ISSN: $1130-3743$

\title{
MODELOS DOCENTES DE LOS PROFESORES UNIVERSITARIOS
}

\author{
Teaching Models of University Professors
}

\section{Modèles d'enseignement des professeurs universitaires}

Bernardo Gargallo López*, Amparo FernáNDez MarCH* y Miguel Ángel JiméNEZ RODRÍGUEZ**

* Universidad de Valencia. Departamento de Teoría de la Educación. Facultad de Filosofía y Ciencias de la Educación. Avda. Blasco Ibáñez, 30. 46010 Valencia. Correo-e: bernardo.gargallo@uv.es, afernama@ice.upv.es

** Universidad Católica de Valencia. Facultad de Ciencias de la Educación y del Deporte Edetania. Campus de Valencia-Santa Úrsula (Guillem de Castro, 94). 46003 Valencia. Correo-e: mangel.jimenez@ucv.es

Fecha de recepción: enero de 2007

Fecha de aceptación definitiva: abril de 2007

BIBLID [(1130-3743) 19, 2007, 167-189]

\section{RESUMEN}

El objetivo fundamental de este trabajo era precisar los modelos docentes de los profesores universitarios para corroborar si se ajustaban a los requerimientos del espacio europeo de educación superior (que preconiza un modelo centrado en el aprendizaje con dominio de competencias pedagógicas). Encontramos dos modelos, uno centrado en el aprendizaje y otro centrado en la enseñanza, y otros dos modelos intermedios. Un grupo de profesores (alrededor de un $48 \%$ de la muestra de 326 profesores) se encuadraba en el modelo centrado en el aprendizaje, de corte constructivista, y se subdividía en dos grupos, uno de ellos más centrado en el aprendizaje y con más habilidades docentes. También encontramos otro grupo (alrededor del $52 \%$ de los profesores), centrado en la enseñanza y que utilizaba metodologías tradicionales. Este grupo también se subdividía en otros dos, uno más tradicional y 
con menos habilidades docentes que el otro. Estos resultados reclaman la atención de los gestores universitarios y el diseño de ofertas racionales de formación que ayuden a los profesores a adquirir las competencias pedagógicas necesarias.

Palabras clave: modelos de enseñanza y evaluación, modelo centrado en el aprendizaje, modelo centrado en la enseñanza, profesores universitarios, educación superior.

\section{SUMMARY}

The main objective of this work was to specify the teaching models of university professors in order to find out whether they meet the requirements of the European higher education area (which upholds a learning-centred model with pedagogical competencies). We found two models, one of them learning-centred and the other one teaching-centred, with two intermediate models. A group of professors (around $48 \%$ of the sample of 326 professors) fitted with the constructivist learning-centred model, and this group was subdivided into two, one of them more learningcentred and with more teaching and assessment abilities than the other. We also found another group (around 52\% of the professors), teaching-centred and which used traditional methodologies. This group was also subdivided into two, one of them more traditional and with less teaching and assessment abilities than the other. These results demand the attention of the university managers and also the design of rational offers of training that help the professors to acquire the necessary pedagogical competencies.

Key words: teaching and assessment models, learning-centered model, teachingcentered model, university professors, higher education.

\section{SOMMAIRE}

L'objectif fondamental de ce travail était de préciser les modèles d'enseignement des professeurs universitaires pour corroborer s'ils étaient adaptés ou pas aux exigences de l'espace européen d'éducation supérieur (qui préconise un modèle centré sur l'apprentissage avec la maîtrise de compétences pédagogiques). Quatre modèles ont été trouvés. L'un est centré sur l'apprentissage, l'autre sur l'enseignement, avec deux modèles intermédiaires. Un groupe de professeurs (autour de $48 \%$ d'un échantillon de 326 professeurs) était encadré dans le modèle centré sur l'apprentissage, de type constructiviste et subdivisé en deux sous-groupes, l'un plus centré sur l'apprentissage et possédant des habilités d'enseignement et d'évaluation plus poussées que l'autre. Un autre groupe de.professeurs a été trouvé (environ $52 \%$ de l'échantillon), centré sur l'enseignement et utilisant des méthodologies traditionnelles. Ce groupe était aussi subdivisé en deux sous-groupes, l'un plus traditionnel et avec moins d'habilités d'enseignement que l'autre. Les dirigeants universitaires devraient tenir compte de ces résultats et l'offre rationnelle 
BERNARDO GARGALLO LÓPEZ, AMPARO FERNÁNDEZ MARCH Y MIGUEL ÁNGEL JIMÉNEZ RODRÍGUEZ MODELOS DOCENTES DE LOS PROFESORES UNIVERSITARIOS

de formation des professeurs devrait viser l'acquisition de ces compétences pédagogiques nécessaires.

Mots clés: modèles d'enseignement et d'évaluation, modèle centré dans l'apprentissage, modèle centré dans l'enseignement, professeurs universitaires, enseignement supérieur.

\section{INTRODUCCIÓN}

El interés por la enseñanza superior como campo de investigación es relativamente reciente y toma cuerpo como tal a mediados del siglo XX. Las razones para este retraso son diversas. Por un lado, las facultades y centros de investigación en educación, que son los centros especializados en investigación educativa, se ocupaban más bien de la educación primaria y secundaria. Su interés por la educación más avanzada era secundario y periférico. Por otro, se trata de un campo interdisciplinar, lo que siendo una indudable fortaleza se traduce muchas veces también en debilidad, habida cuenta de la dificultad de constituir grupos interdisciplinares sólidos que investiguen en este campo y de arbitrar la metodología pertinente para la investigación. Además, hasta hace poco tiempo no ha habido una gran demanda de datos e investigación por los usuarios potenciales (universidades, administración educativa, gobiernos, etc.). Ello explica el reducido número de investigadores especializados en la educación superior y la dificultad de la emergencia de un campo específico de investigación (Altbach, 2002). Así y todo, a partir de los años 50 se va desarrollando un cuerpo importante de investigación y hoy es un campo tan sólido como lo son otros campos educativos tradicionales. Diversas razones explican el cambio: la preocupación por la calidad de las instituciones de educación superior (Morris, 2003), la necesidad de datos por parte de los gobiernos para la planificación, la expansión de las propias instituciones de educación superior, el desarrollo de investigación institucional de los propios centros, la creación de centros específicos de investigación, de agencias estatales, de redes de investigadores, la incidencia de organizaciones internacionales (UNESCO, OCDE...), etc. Hay diversos ámbitos de investigación que gozan ya de una cierta tradición en este campo: así la economía de la educación superior, los estudios comparados, la política educativa, la administración de la educación, los procesos de enseñanza y aprendizaje, etc.

El excelente trabajo de revisión de Altbach (2002) confirma que, entre los diversos ámbitos que se investigan en el campo, la temática relativa a los procesos de enseñanza y aprendizaje goza actualmente de un interés creciente y se contempla como un elemento sustancial de la calidad de las instituciones de educación superior. En este ámbito concreto se ubica el trabajo que presentamos, que tiene como objetivo fundamental precisar los modelos de enseñanza y evaluación de los profesores universitarios y sus perfiles en cuanto concepción del conocimiento, de 
la enseñanza y el aprendizaje, a modalidades de enseñanza, evaluación y a habilidades docentes.

Si siempre ha tenido interés la realización de este tipo de trabajos, hay razones contextuales que en el momento actual lo incrementan de modo sustancial. Estamos inmersos en un proceso de convergencia, en la educación superior de los países de la Unión Europea, que comporta, entre otros aspectos, reconfigurar los papeles de los profesores y de los alumnos. Las teorías del aprendizaje vigentes en los documentos para la convergencia en la universidad preconizan una pedagogía universitaria más centrada en el aprendizaje que en la enseñanza (Samuelowicz y Bain, 2001). Ello supone un cambio en el papel del profesor, que debe actuar como mediador, como diseñador de entornos de aprendizaje, como propiciador del aprendizaje autónomo de los alumnos, lo que exige claramente competencias pedagógicas, manejo de técnicas y de recursos educativos, frente a modelos tradicionales centrados en el puro dominio de los contenidos y en su exposición ante los alumnos, basados en una concepción del profesor como poseedor y dispensador del saber de su disciplina, que se limita básicamente a la transmisión de los conocimientos.

Evidentemente, este planteamiento exige un cambio, también en el papel del alumno, que no puede limitarse a ser el receptor y reproductor de los conocimientos transmitidos por el profesor, sino un sujeto activamente implicado en el proceso de aprendizaje, que debe indagar, cuestionar, elaborar, investigar, realizar aportaciones personales, etc.

En ese contexto, es fundamental conocer cuáles son las concepciones de los profesores universitarios en torno a la enseñanza y precisar sus perfiles de enseñanza y evaluación así como sus habilidades docentes, de cara a determinar en qué medida se ajustan o no a los nuevos requerimientos de la enseñanza universitaria. Como dijimos antes, ése es, pues, el objetivo de este trabajo que aquí presentamos. Ello permitirá a los gestores universitarios tomar las medidas oportunas para poner en marcha procesos de formación de profesores, cuando sea preciso, para las nuevas coordenadas definidas por la convergencia.

\section{El ESTADO DE LA CUESTIÓN}

Enseñanza y aprendizaje son dos caras de la misma moneda, y la concepción del aprendizaje de los profesores tiene mucho que ver con su concepción de la enseñanza y con el modo de abordarla.

Una concepción del aprendizaje como adquisición o incremento de conocimientos, como reproducción de lo ya dado, suele ir ligada a una concepción de la enseñanza como transmisión de información.

Por el contrario, una concepción del aprendizaje como proceso de construcción del conocimiento, que supone comprensión significativa y que comporta cambios conceptuales y personales, suele ir ligada a una concepción de la enseñanza como facilitación del aprendizaje. 
Diversas investigaciones realizadas sobre las creencias y convicciones de los profesores con respecto a la enseñanza y el aprendizaje de los estudiantes, y sobre lo que los profesores dicen que hacen cuando enseñan, conducen a dos grandes modelos: el modelo de transmisión del conocimiento o modelo centrado en la enseñanza, también denominado modelo centrado en el profesor y el modelo de facilitación del aprendizaje, o modelo centrado en el aprendizaje, que también se denomina modelo centrado en el alumno. Ambos serían los extremos de un continuum en el que se ubicarían categorías intermedias (Kember, 1997; Samuelowicz y Bain, 2001).

Para precisar el estado de la cuestión, recogemos referencias de las investigaciones aludidas, que pueden considerarse básicas en el tema y que han sido realizadas utilizando métodos cualitativos prioritariamente -la mayoría de ellas con un enfoque denominado fenomenográfico-1:

Larsson (1983) encontró dos modelos, basados en dos concepciones de la enseñanza de los profesores: de "transmisión de información" y de "facilitación del aprendizaje".

Dall'Alba (1991) halló tres modelos: "transmisión del conocimiento" (que incluía dos categorías: "presentación de información" y "conexión de la teoría con la práctica"), "facilitación del aprendizaje" (que incluía también dos categorías: "exploración de caminos para la comprensión", y "provocando el cambio conceptual"), y entre ambos uno intermedio, con una categoría: "desarrollo de conceptos".

Samuelowicz y Bain (1992) encontraron tres modelos de enseñanza: "transmisión del conocimiento" (con dos categorías: "dar información" y "transmitir el conocimiento"), "facilitación del aprendizaje" (con dos categorías: "cambiar las concepciones de los estudiantes" y "apoyar el aprendizaje de los estudiantes"), y entre ambos uno intermedio, con una categoría, "facilitación de la comprensión".

Martin y Ramsden (1992) encontraron también tres modelos: "transmisión del conocimiento" (con dos categorías: "presentación del contenido" y "organización del contenido o proceso"), "facilitación del aprendizaje" (con una categoría: "facilitación de la comprensión mediante el enganche con contenido y proceso"), y entre ambos uno intermedio, con una categoría: "organizar el entorno de aprendizaje".

Gow y Kember (1993) y Kember y Gow (1994) encontraron dos modelos en los profesores: "transmisión de información" y "facilitación del aprendizaje".

Kember (1997), que realizó un trabajo de síntesis de literatura sobre el tema, analizando trece investigaciones realizadas, encontró también tres modelos: «transmisión del conocimiento/orientación centrada en el profesor/centrada en el contenido" (con dos categorías: "impartición de información" y "transmisión de la

1 En este enfoque, que es interpretativo, se ha utilizado habitualmente la entrevista con los profesores de la universidad para recoger información sobre sus concepciones en torno al conocimiento, al aprendizaje, a la enseñanza, a la relación entre enseñanza-aprendizaje, etc. En ocasiones también se les ha pedido que describan situaciones concretas de enseñanza que den ejemplo de sus perspectivas. Posteriormente se han categorizado los resultados obtenidos. 
estructura del conocimiento"), "facilitación del aprendizaje/orientación centrada en el aprendizaje/centrada en el estudiante" (con dos categorías: "facilitación de la comprensión" y "cambio conceptual/desarrollo intelectual"), y entre ambos uno intermedio, con una sola categoría: «interacción estudiante-profesor".

Especial mención merece la investigación de Samuelowicz y Bain (2001) en que los autores revisan la literatura y evalúan y refinan los hallazgos de un trabajo previo (Samuelowicz y Bain, 1992). En este caso utilizaron una muestra de 39 profesores universitarios de tres universidades de Brisbane (Australia) de diversas especialidades, haciendo uso de entrevistas semiestructuradas. Los autores utilizaron análisis cualitativo y también realizaron clusters (conglomerados) jerárquicos a partir de este análisis. Para ello asignaron previamente valores cuantitativos a las categorías halladas en el análisis cualitativo. Los resultados cuantitativos confirmaron los cualitativos.

Samuelowicz y Bain (2001) encontraron dos grandes orientaciones o modelos: una orientación centrada en la enseñanza y otra orientación centrada en el aprendizaje.

Cada una de esas dos orientaciones incluía varias dimensiones relativas a las creencias de los profesores.

La orientación centrada en la enseñanza integraba tres dimensiones, de más a menos transmisivas: la primera, "impartición de información", la segunda, "transmisión de conocimiento estructurado", y la tercera, de "promoción y facilitación de la comprensión".

La orientación centrada en el aprendizaje incluía cuatro dimensiones, siendo la última la más comprometida con la orientación: la primera, "ayuda a los estudiantes para hacerse expertos", la segunda, "prevención de errores", la tercera, "negociación de significados", y la cuarta, "promoviendo la creación de conocimiento".

Los autores replantean, pues, su posición inicial (Samuelowicz y Bain, 1992) ya que en la investigación anterior proponían tres orientaciones o modelos, con uno intermedio entre el modelo de "transmisión" y el de "facilitación", al que denominaron "facilitador de la comprensión". En el trabajo de 2001 no encuentran ninguna evidencia empírica de que exista tal modelo u orientación intermedio. Los resultados del estudio más reciente parecen más sólidos tanto por haber trabajado con una muestra mayor (39 profesores en 2001 frente a 13 en 1992) como por el tratamiento de los datos, más sofisticado en 2001.

Una síntesis de los dos modelos (Samuelowicz y Bain, 2001) es la que sigue:

La orientación centrada en la enseñanza busca como producto del aprendizaje la reproducción. El uso que se espera de lo aprendido es para aprobar o para usarlo en el futuro. La responsabilidad de la organización o transformación del conocimiento es del profesor. El conocimiento se entiende como algo construido externamente, por otros. Las concepciones del estudiante no se tienen en cuenta. La interacción entre el estudiante y el profesor es mínima y unidireccional o como mucho bidireccional para mantener la atención o para asegurarse de la comprensión y aclarar dudas. El control del contenido rećae sobre el profesor. No se promueve el desarrollo profesional. El interés y la motivación debe promoverlos el profesor. 
La orientación centrada en el aprendizaje busca como producto del aprendizaje el cambio mental. El uso que se espera de lo aprendido es para la vida y para interpretar la realidad. La responsabilidad de la organización o transformación del conocimiento es compartida o de los estudiantes. El conocimiento se entiende como algo construido por los alumnos de modo personal. Las concepciones del estudiante se usan como base para prevenir errores y para promover el cambio conceptual. La interacción entre el estudiante y el profesor es bidireccional para negociar significados. El control del contenido recae sobre el profesor y los estudiantes. Se enfatiza el desarrollo profesional. El interés y la motivación recaen sobre todo en los propios estudiantes.

Un trabajo posterior de Samuelowicz y Bain (2002) corrobora la existencia de dos grandes orientaciones que también se dan en la evaluación, la que pone el énfasis en la reproducción del conocimiento y la que lo hace en su construcción y/o transformación.

Desde nuestro punto de vista la orientación centrada en el aprendizaje es claramente constructivista, tanto por la concepción del conocimiento que sustenta como por la concepción del aprendizaje, de la enseñanza y de la evaluación, aunque los investigadores no le adjudiquen esta denominación.

A partir de estos trabajos de investigación, suscribimos la concepción que establece dos modos fundamentales de abordar la docencia en la universidad, que dan origen a dos modelos comúnmente aceptados hoy: el modelo "centrado en la enseñanza" (modelo tradicional, centrado en el profesor, de transmisión de información, expositivo) y el modelo "centrado en el aprendizaje" (modelo constructivista ${ }^{2}$, centrado en el alumno, de facilitación del aprendizaje), siendo conscientes de que seguramente muchos profesores se ubicarán en una "zona intermedia" entre los dos extremos.

Fundamentándonos en las investigaciones aludidas (Dall'Alba, 1991; Gow y Kember, 1993; Kember, 1997; Kember y Gow, 1994; Larsson, 1983; Samuelowicz y Bain, 2001 y 2002), en otras que también hemos analizado (Alonso y Méndez, 1999; Biggs, 2005; García Valcárcel, 1993³; Monereo y Pozo, 2003) y en la propia reflexión, las características que para nosotros acotan ambos modelos son las que siguen:

\footnotetext{
${ }^{2}$ En este sentido, nosotros, que compartimos algunos de los elementos de la teoría constructivista piagetiana, estamos más cerca del enfoque socio-cultural (LEONTIEv, 1978; LuRIA, 1987; VYGOTSKY, 1978) y de la teoría de la cognición situada (Bereiter, 1997; ENGELSTRÖM y COLE, 1997; LAVE, 1997; Rogoff, 1993; WENGER, 2001), con su énfasis en el diseño de entornos de aprendizaje que, trabajando sobre atareas auténticas", propicien la construcción de significados en interacción con los otros con el soporte de la mediación del profesor universitario, habida cuenta de que la universidad es el ámbito en que nos ubicamos.

${ }^{3}$ Esta investigadora recogió datos de 166 profesores universitarios de la Universidad de Cantabria y de sus asignaturas y de 1.987 alumnos que las cursaban, pasando un cuestionario sobre conductas docentes a los alumnos y otro sobre satisfacción personal, interés y dedicación a la docencia y formación pedagógica a los profesores. Posteriormente realizó análisis de clusters con los datos del cuestionario de los alumnos y encontró dos modelos de enseñanza, el expositivo, que coincide sustancialmente 


\subsection{Modelo centrado en la enseñanza/tradicional/centrado en el profesor/ transmisor de información}

Concepción del conocimiento

El modelo centrado en la enseñanza entiende el conocimiento como algo construido externamente. Existe un corpus de conocimientos científicos acotado por la disciplina y elaborado por grandes pensadores, que hay que transmitir ( $1 \mathrm{a}$ rueda ya está descubierta") y que posee el profesor. Es él quien tiene la responsabilidad de organizar y transformar el conocimiento.

\section{Concepción del aprendizaje}

De modo coherente con la concepción del conocimiento, el aprendizaje se entiende como adquisición o incremento de conocimientos, que el estudiante utilizará en la propia disciplina y, en todo caso, en el futuro.

Concepción de la enseñanza y papel del profesor

La enseñanza se entiende como transmisión de conocimientos operada por el profesor, que es el que sabe. Lo más importante, pues, para ser un buen profesor, es dominar la materia que se imparte, estar al día y explicar bien. El buen profesor conoce la materia, transmite bien la información y facilita su comprensión a los alumnos.

Metodología docente

De modo coherente con estas concepciones, se utiliza como método básico, si no exclusivo, la lección magistral y la exposición del profesor. En esta metodología se potencia poco la interacción profesor-alumnos, que se limitan en general a escuchar y copiar. La interacción del profesor con los alumnos es preferentemente unidireccional: el profesor explica, pone buenos ejemplos, etc., para que los estudiantes comprendan la materia. Cuando es bidireccional se orienta sobre todo a mantener la atención del estudiante y a facilitar su comprensión de la materia, limitándose generalmente a responder a preguntas o dudas que plantee el estudiante. No se tienen en cuenta las concepciones del estudiante para prevenir errores o para negociar significados. De hecho, suele faltar el feed-back necesario con respecto a la comprensión de la materia por parte de los estudiantes.

con el modelo centrado en la enseñanza (modelo de transmisión de información), y el interactivo, que incluye bastantes elementos del modelo centrado en la enseñanza. 
Materiales de aprendizaje

Se utilizan como materiales de estudio preferentemente apuntes del profesor y/o un libro de texto.

Metodología de evaluación

La metodología de evaluación correspondiente utiliza el examen como método de evaluación, preferentemente con preguntas cerradas que exigen uredecir", repetir lo aprendido y/o pruebas objetivas. El criterio fundamental para aprobar es que los alumnos sean capaces de reproducir los conocimientos adquiridos.

Uso de la tutoría

La tutoría se utiliza como un periodo temporal en que el profesor está en su despacho atendiendo a los alumnos que desean hacer uso de sus servicios.

\subsection{Modelo centrado en el aprendizaje/constructivista/centrado en el alumno/ facilitador del aprendizaje}

Concepción del conocimiento

El modelo centrado en el aprendizaje entiende el conocimiento no como algo que es fijo e inmutable, que está ahí fuera para llenar el vacío de la ignorancia del estudiante, sino como una construcción social y negociada. La responsabilidad de organizar y transformar el conocimiento es del profesor y del alumno.

\section{Concepción del aprendizaje}

El aprendizaje se contempla como un proceso de construcción personal, compartido y negociado con otros, que comporta la comprensión significativa y que da lugar a cambios conceptuales y personales. Los conocimientos adquiridos deben ser útiles para algo más que para aprobar la disciplina o para el futuro, han de servir al estudiante para interpretar la realidad en que está inmerso.

Concepción de la enseñanza y papel del profesor

La enseñanza se concibe como un proceso interactivo que debe facilitar la construcción personal del conocimiento. El papel del profesor es el de facilitador del aprendizaje del estudiante, no transmisor de información. Tan importante como conocer la materia, para ser buen profesor, lo es disponer de formación didáctico- 
pedagógica (sobre diseño instruccional, metodología, teorías del aprendizaje...) que capacite al profesor para diseñar entornos ricos de aprendizaje.

\section{Metodología docente}

De modo coherente con estos planteamientos, se hace uso de diversos métodos en función de los objetivos y del contexto. El profesor busca la implicación del estudiante para potenciar la comprensión, y fomenta su desarrollo personal, su autonomía y la mejora de su competencia para aprender a aprender. Se trata de que el estudiante llegue a ser un aprendiz independiente y de que aprenda a autoevaluar competentemente su trabajo. La interacción del profesor con los estudiantes es preferentemente bidireccional: se tienen en cuenta las concepciones del estudiante y se busca comprobar su comprensión de los contenidos de aprendizaje y promover la negociación de significados. Así, los métodos expositivos se complementan con métodos interactivos -se utiliza el diálogo y las preguntas, se hace uso de técnicas de grupo (trabajo cooperativo, discusión en grupo, role-playing, etc.)- para potenciar una interacción más rica y útil para el aprendizaje. Los métodos interactivos no se utilizan como puros recursos para romper la monotonía de la clase o para innovar sin más, sino como procedimientos que faciliten la negociación de significados y la reconstrucción del conocimiento. Se pueden usar también, en función del tiempo disponible y del nivel de los estudiantes, métodos de indagación-investigación -con diversas técnicas: seminarios, trabajos de investigación, individuales y en equipo, solución de problemas, estudio de casos, simulaciones, etc.-.

\section{Materiales de aprendizaje}

Se utilizan diversos materiales para el aprendizaje de cara a que el estudiante sintetice información, la elabore, la critique, etc. (manuales, apuntes, artículos, monografías, artefactos, materiales audiovisuales, etc.).

Cuando se usan las nuevas tecnologías no se utilizan sólo como vehículos de depósito de información, sino para potenciar la interacción y el trabajo cooperativo...

\section{Metodología de evaluación}

La metodología de evaluación correspondiente tiene un enfoque formativo. El profesor puede utilizar exámenes pero con formatos que van más allá de la reproducción, por ejemplo con resolución de problemas y estudio de casos o simulaciones que exigen la reelaboración y aplicación de lo aprendido - no basta con redecir lo aprendido- pero suele valorar también otros trabajos realizados por el alumno durante el curso, dando información a los alumnos de sus progresos y deficiencias para su corrección, o bien utiliza procedimientos alternativos sin exámenes, de tipo procesual 
y formativo -portafolios, contrato pedagógico, seguimiento individualizado y continuo, etc.- que permiten valorar en qué medida el estudiante ha realizado una construcción personal pertinente de los conocimientos. El feed-back se considera sustancial y se ofrece al estudiante para que aprenda a autoevaluar su progreso.

Uso de la tutoría

La tutoría se usa de modo activo y sistemático, para asesorar a los estudiantes, no limitándose a esperar a que acudan los que lo deseen, planificando su utilización.

\section{LA INVESTIGACIÓN. METODOLOGÍA Y PROCEDIMIENTO}

Los resultados que aquí presentamos se inscriben en el contexto de una investigación de tres años ${ }^{4}$, que pretende, entre otros objetivos, valorar en qué medida influyen los métodos de enseñanza y evaluación de los profesores universitarios en los modos de aprender de los estudiantes. Se trata, en un primer momento, con datos del primer y segundo año, de corroborar a nivel empírico la existencia de los dos modelos teóricos presentados en el apartado anterior, y de precisar los perfiles de enseñanza y evaluación de los profesores universitarios integrados en los mismos, como hemos reflejado en la introducción.

\subsection{Instrumentos de medida}

Al no disponer de un instrumento de evaluación ajustado a los objetivos de la investigación, elaboramos y validamos un cuestionario propio (el CEMEDEPU, Cuestionario de Evaluación de la Metodología Docente y Evaluativa de los Profesores Universitarios), apoyándonos en trabajos previos de otros investigadores (Dall'Alba, 1991; García Valcárcel, 1993; Gow y Kember, 1993; Kember, 1997; Kember y Gow, 1994; Martin y Ramsden, 1992; Samuelowicz y Bain, 2001) y en nuestra propia reflexión.

El cuestionario CEMEDEPU se diseñó como un cuestionario formado por tres escalas y, una vez validado, acabó constituido por 51 ítems. Las dos primeras escalas se basan en la estructura teórica subyacente de los dos modelos, descrita en los apartados anteriores (apartados 2.1 y 2.2). La tercera incorpora

${ }^{4}$ Se trata de la investigación "Estrategias de enseñanza y estrategias de aprendizaje en la universidad. Análisis de la incidencia de variables fundamentales en los modos en que los alumnos afrontan el aprendizaje", aprobada por el Ministerio de Ciencia y Tecnología de España por medio de convocatoria pública de tipo competitivo, y financiada por el Ministerio de Ciencia y Tecnología y por el FEDER (Fondo Europeo de Desarrollo Regional), que es dirigida por el profesor Bernardo Gargallo (código SEC2003-06787/PSCE). 
referencia de las habilidades docentes que podemos considerar fundamentales en el profesor universitario.

La primera de las escalas, formada por 16 ítems, evalúa los componentes fundamentales del modelo centrado en la enseñanza (concepción del conocimiento, del aprendizaje, de la enseñanza, papel del profesor en este modelo, metodología de enseñanza, materiales de aprendizaje y metodología de evaluación coherente con una concepción tradicional).

La segunda, formada por 17 ítems, evalúa los componentes fundamentales del modelo centrado en el aprendizaje (concepción del conocimiento, del aprendizaje, de la enseñanza, papel del profesor en este modelo, metodología de enseñanza, materiales de aprendizaje y metodología de evaluación coherente con una concepción constructivista).

La tercera, formada por 18 ítems, evalúa las habilidades docentes del profesor eficaz: planificación, comunicación con los alumnos, metodología adecuada a los objetivos, evaluación coherente con los mismos y con criterios claros, etc.

El cuestionario adopta el formato de las escalas tipo Likert con cinco opciones de respuesta para cada ítem, que oscilan desde "muy en desacuerdo" hasta "muy de acuerdo".

El instrumento fue validado con una muestra de 232 profesores de las dos universidades públicas de la ciudad de Valencia (España), la Universidad de Valencia/Estudi General (UVEG), y la Universidad Politécnica de Valencia (UPV). Previamente llevamos a cabo un muestreo representativo de los profesores de ambas universidades, equilibrado proporcionalmente para ambas, con un nivel de confianza del 95\% y con un error máximo del 5\%.

El cuestionario resultante de la validación logró un alto nivel de consistencia interna: el coeficiente alfa de Cronbach para la primera escala fue ,879, para la segunda ,832, y para la tercera ,839.

\subsection{Muestra}

A la muestra inicial de 232 profesores ya aludidos hay que sumar 93 profesores de las tres universidades de la ciudad de Valencia, las dos públicas: Universidad de Valencia/Estudi General (UVEG), Universidad Politécnica de Valencia (UPV) y una tercera privada, la Universidad Católica de Valencia (UCV) ${ }^{5}$, seleccionados de diversas facultades y titulaciones de las mismas de cara a configurar un grupo de profesores lo suficientemente variado con diferentes perfiles de docencia y evaluación que formaron parte del trabajo desarrollado en el segundo año del proyecto.

${ }^{5}$ La tercera de las universidades de la ciudad de Valencia, la UCV, una universidad privada como reflejamos arriba, se sumó al proyecto en su segundo año (2004-2005), a partir de su constitución oficial como universidad en el año 2004.

(C) Ediciones Universidad de Salamanca

Teor. educ. 19, 2007, pp. 167-189 
Por tanto, la muestra de la que exponemos los datos aquí reseñados ha sido de 326 profesores de las tres universidades.

\subsection{Procedimiento}

Los profesores contestaron el cuestionario CEMEDEPU contextualizándolo en una asignatura que impartían en el curso correspondiente (232 de ellos en 20032004 y 93 en 2004-2005). Los resultados obtenidos fueron sometidos a los análisis estadísticos pertinentes para delimitar la concreción de modelos de enseñanza y evaluación, concretados en grupos de profesores y en sus perfiles.

\section{Resultados Y SU Discusión. MODELOS, GRUPOS Y PERFILES}

Para la concreción de los modelos y de sus perfiles, utilizamos como variables las puntuaciones obtenidas por los profesores en los 51 ítems de las tres escalas que conformaban el cuestionario y llevamos a cabo análisis de conglomerado o clusters:

$\mathrm{El}$ análisis de conglomerados permite identificar grupos de profesores atendiendo a las relaciones entre las puntuaciones de los ítems, lo que nos da pie a obtener una imagen simplificada de la realidad, concretada en los diferentes modelos y en los perfiles de sus profesores a partir de la información obtenida de cada profesor concreto. De este modo, dispondremos de información relevante que nos permita realizar reflexiones y recomendaciones precisas y matizadas que puedan ser de utilidad en el establecimiento de políticas sobre el tema que nos ocupa.

A tal efecto, hemos utilizado la técnica de análisis de conglomerados de $k$ medias. Este procedimiento intenta identificar grupos de casos relativamente homogéneos basándose en las características seleccionadas y utilizando un algoritmo que puede gestionar un gran número de casos. El algoritmo requiere que el investigador especifique el número de conglomerados y que seleccione el más pertinente utilizando diversos indicadores.

Se han explorado opciones entre 3 y 6 agrupamientos. La solución de cuatro grupos es la más adecuada dado que en ella todos los ítems son significativos para la clasificación, admite una interpretación coherente y parsimónica, y evita agrupamientos excesivamente reducidos. Los centros de los conglomerados aparecen en la Tabla 1 y el número de casos de cada conglomerado en la Tabla 2. 
TABLA 1

CENTROS DE LOS CONGLOMERADOS FINALES

\begin{tabular}{|c|c|c|c|c|}
\hline \multirow[t]{2}{*}{ Ítems } & \multicolumn{4}{|c|}{ Conglomerado } \\
\hline & 1 & 2 & 3 & 4 \\
\hline $\begin{array}{l}\text { 1. Los conocimientos científicos se hallan establecidos en las } \\
\text { profesores los que disponen de ellos para enseñarlos a los }\end{array}$ & 2,82 & 3,61 & 2,67 & 3,46 \\
\hline $\begin{array}{l}\text { 2. Basta con que los alumnos aprendan y comprendan los contenidos científicos } \\
\text { fundamentales de la disciplina; no necesitan ir más allá en su formación universitaria }\end{array}$ & 1,45 & 2,24 & 1,51 & 2,36 \\
\hline 3. Aprender es incrementar los conocimientos disponibles & 3,25 & 3,77 & 2,42 & 3,36 \\
\hline $\begin{array}{l}\text { 4. El trabajo esencial del profesor universitario es transmitir los conocimientos a sus } \\
\text { alumnos }\end{array}$ & 2,58 & 3,73 & 2,09 & 3,35 \\
\hline 5. Lo más importante para ser buen profesor es dominar la materia que se imparte & 2,71 & 3,42 & 2,40 & 3,31 \\
\hline 6. Un buen profesor es el que explica bien su asignatura & 3,25 & 3,89 & 2,70 & 3,73 \\
\hline $\begin{array}{l}\text { 7. Mi responsabilidad fundamental es organizar bien los conocimientos que deben } \\
\text { aprender los alumnos y presentarlos de modo comprensible }\end{array}$ & 3,77 & 4,27 & 3,09 & 3,96 \\
\hline $\begin{array}{l}\text { 8. El tiempo de las clases teóricas debe usarse para explicar bien los contenidos de } \\
\text { la asignatura }\end{array}$ & 3,45 & 4,06 & 3,02 & 3,99 \\
\hline 9. En mis clases teóricas la lección magistral es la metodología fundamental & 2,16 & 3,42 & 2,85 & 3,81 \\
\hline 10. El papel básico de los alumnos en clase es estar atentos y tomar bien los apuntes & 1,66 & 2,34 & 1,70 & 2,65 \\
\hline $\begin{array}{l}\text { 11. Las intervenciones de los alumnos en clase deben ser } p \\
\text { contestar a las preguntas del profesor o para plantear las du }\end{array}$ & 2,43 & 3,32 & 2,20 & 3,29 \\
\hline 12. El mejor método para evaluar a los alumnos es el examen & 1,83 & 2,95 & 2,11 & 3,27 \\
\hline limitarse a la valoración de los conocimien & 1,90 & 2,96 & 2,11 & 3,18 \\
\hline sultados del aprendizaje del & 3,37 & 4,01 & 3,13 & 3,90 \\
\hline 15. Sólo el profesor está capacitado para valorar los aprendizajes de los estudiantes & 2,33 & 3,27 & 2,33 & 3,12 \\
\hline $\begin{array}{l}\text { 16. El criterio fundamental para aprobar a los alumnos es que hayan aprendido los } \\
\text { conocimientos trabajados en la asignatura y que sean capaces de reproducirlos } \\
\text { de manera clara }\end{array}$ & 2,49 & 3,93 & 2,51 & 3,60 \\
\hline $\begin{array}{l}\text { 17. El conocimiento no es algo establecido en las disciplinas y recogido en los } \\
\text { manuales y otros documentos, sino algo a construir entre estudiantes y profesores }\end{array}$ & 4,68 & 3,83 & 3,92 & $3, \mathbf{4 4}$ \\
\hline 18. El conocimiento debe ser construido por los estudiantes con ayuda del profesor & 4,53 & 3,78 & 3,89 & 3,32 \\
\hline 19. Aprender es construir personalmente significados & 4,45 & 3,94 & 3,94 & 3,51 \\
\hline $\begin{array}{l}\text { 20. Doy a los estudiantes oportunidad de realizar aportaciones personales; por ejemplo, } \\
\text { les pido que predigan resultados, que propongan hipótesis y las comprueben, etc. }\end{array}$ & 4,68 & 4,26 & 4,09 & 3,49 \\
\hline $\begin{array}{l}\text { 21. Los conocimientos que mis estudiantes adquieren les sirven ya para interpretar } \\
\text { la realidad en que están inmersos, no sólo para aprobar la materia } \\
\end{array}$ & 4,58 & 4,30 & 3,97 & 3,95 \\
\hline $\begin{array}{l}\text { 22. Dispongo mi clase como un entorno de aprendizaje que moviliza el aprendizaje } \\
\text { activo del alumno (a través del planteamiento y resolución de problemas, del fomento } \\
\text { de la participación del estudiante, del establecimiento de conexiones con la realidad) }\end{array}$ & 4,68 & 4,34 & 3,92 & 3,55 \\
\hline $\begin{array}{l}\text { 23. Adopto una metodología de enseñanza variada y complementaria que adapto a } \\
\text { las características del grupo de alumnos }\end{array}$ & 4,55 & 4,10 & 3,63 & 3,04 \\
\hline $\begin{array}{l}\text { 24. Hago uso de la pregunta en mi clase de manera sistemática para ayudar a pensar } \\
\text { a los estudiantes }\end{array}$ & 4,68 & 4,34 & 4,14 & 3,90 \\
\hline
\end{tabular}




\begin{tabular}{|c|c|c|c|c|}
\hline & 1 & 2 & 3 & 4 \\
\hline $\begin{array}{l}\text { 25. Hago uso del estudio de casos y/o simulaciones en clase para potenciar la } \\
\text { integración de la teoría y la práctica }\end{array}$ & 4,50 & 4,25 & 3,78 & 3,73 \\
\hline 26. Realizo seminarios con los estudiantes de mi asignatura & 3,95 & 3,09 & 2,57 & 1,90 \\
\hline 27. Muestro aplicaciones de la teoría a los problemas reales & 4,71 & 4,49 & 4,13 & 4,10 \\
\hline $\begin{array}{l}\text { 28. Utilizo la tutoría con un plan de trabajo establecido para asesorar a los alumnos } \\
\text { y no me limito a esperar a que acudan los que lo deseen }\end{array}$ & 3,91 & 2,90 & 2,58 & 1,91 \\
\hline $\begin{array}{l}\text { 29. El uso que hago de las nuevas tecnologias fomenta la participación de los alumnos, la } \\
\text { interactividad, la cooperación, etc., mediante la tutoría telemática, foros de discusión, etc. }\end{array}$ & 3,68 & 3,12 & 2,59 & 2,19 \\
\hline $\begin{array}{l}\text { 30. Mis alumnos deben aprender a autoevaluar completamente su trabajo y yo les } \\
\text { ayudo a que lo hagan }\end{array}$ & 4,22 & 3,27 & 3,04 & 2,55 \\
\hline $\begin{array}{l}\text { 31. Complemento el examen como método de evaluación con otros métodos de } \\
\text { orientación formativa/continua (p. ej. trabajos, ensayos, informes, portafolios, etc.) }\end{array}$ & 4,74 & $\mathbf{3 , 8 3}$ & 3,94 & 2,89 \\
\hline $\begin{array}{l}\text { 32. Uso procedimientos de evaluación formativa/continua (p. ej. preguntas de clase, } \\
\text { trabajos, informes, pruebas, ensayos, etc.) revisando y devolviendo corregidos a } \\
\text { los alumnos los trabajos escritos con instrucciones para la mejora }\end{array}$ & $4, \mathbf{4 1}$ & 3,55 & 3,72 & 2,64 \\
\hline $\begin{array}{l}\text { 33. Evalúo no sólo para valorar los resultados del alumno sino para obtener } \\
\text { información del proceso de aprendizaje e introducir las mejoras necesarias }\end{array}$ & 4,71 & 4,09 & 3,89 & 3,29 \\
\hline 34. Planifico mi asignatura todos los cursos dedicando tiempo a esta tarea & 4,70 & 4,40 & 4,13 & 3,95 \\
\hline 35. Facilito a mis alumnos el programa de la asignatura y les informo sobre el mismo & 4,93 & 4,73 & 4,53 & 4,53 \\
\hline 36. Establezco claramente los objetivos de mi asignatura & 4,77 & 4,63 & 4,26 & 4,25 \\
\hline 37. Mis alumnos saben cuáles son las referencias bibliográficas esenciales para l & 4,78 & 4,66 & 4,42 & 4,49 \\
\hline 38. Recuerdo brevemente lo tratado en la clase anterior & 4,49 & 4,47 & 4,24 & 4,21 \\
\hline 39. Al terminar la clase, hago una breve síntesis de lo tratado en ella & 4,17 & 4,01 & 3,72 & $3, \mathbf{4 0}$ \\
\hline 40. Presento los contenidos de manera que promuevan el interés de los alu & 4,57 & 4,27 & 3,95 & 3,78 \\
\hline 41. Procuro transmitir a los alumnos mi interés por la materia que imparto & 4,86 & 4,68 & 4,43 & 4,36 \\
\hline 42. Procuro que en clase exista un clima de buenas relaciones interpersonales & 4,72 & 4,66 & 4,41 & 4,29 \\
\hline 43. Me intereso por los estudiantes como personas & 4,78 & 4,43 & 4,30 & 4,14 \\
\hline 44. Evalúo los aprendizajes de acuerdo con los objetivos establecidos en la planificación & 4,44 & 4,31 & 4,11 & 3,90 \\
\hline $\begin{array}{l}\text { 45. Establezco con claridad los criterios de evaluación de los aprendizajes de los } \\
\text { alumnos y éstos los conocen }\end{array}$ & 4,71 & 4,48 & 4,22 & 4,15 \\
\hline 46. Informo a mis alumnos de los métodos de evaluación que voy a utilizar & 4,84 & 4,66 & 4,48 & 4,45 \\
\hline 47. Mis alumnos conocen los criterios de corrección de las pruebas que utilizo & 4,70 & 4,43 & 4,15 & 4,01 \\
\hline 48. Realizo una evaluación inicial para precisar los conocimientos previos de los alumnos & 3,72 & 2,51 & 2,59 & 1,76 \\
\hline $\begin{array}{l}\text { 49. Evalúo en diferentes momentos del curso para llevar un seguimiento del } \\
\text { aprendizaje de los alumnos }\end{array}$ & 4,31 & 3,35 & 3,27 & 2,40 \\
\hline $\begin{array}{l}\text { 50. Tengo en cuenta los resultados de la evaluación para modificar mi planificación, } \\
\text { metodología y actividad docente a corto o medio plazo }\end{array}$ & 4,46 & 3,83 & 3,69 & 3,26 \\
\hline 51. Oriento a mis alumnos para que mejoren sus resultados & 4,61 & 4,27 & 3,94 & 3,79 \\
\hline
\end{tabular}

Nota: En la columna de la izquierda aparece referencia de la formulación de los 51 ítems del cuestionario. Téngase en cuenta, para la interpretación de la tabla, que las puntuaciones de los centros de los conglomerados finales de cada uno de los grupos -que se recogen en las cuatro columnas de la derecha- se mueven en una escala de puntuación coincidente con las 5 opciones de respuesta a los ítems del cuestionario, siendo $1=$ muy en desacuerdo, $2=$ en desacuerdo, $3=$ indeciso, $4=$ de acuerdo, y $5=$ muy de acuerdo. Hemos marcado en negrita las puntuaciones superiores a 3 , que se mueven desde la indecisión al acuerdo. 
BERNARDO GARGALLO LÓPEZ, AMPARO FERNÁNDEZ MARCH Y MIGUEL ÁNGEL JIMÉNEZ RODRÍGUEZ MODELOS DOCENTES DE LOS PROFESORES UNIVERSITARIOS

TABLA 2

NÚMERO DE CASOS EN CADA CONGLOMERADO

\begin{tabular}{|c|r|r|}
\hline Conglomerados & N..$^{\circ}$ de sujetos & \multicolumn{2}{|c|}{ Porcentaje } \\
\hline 1 & 69,000 & $21,17 \%$ \\
\hline 2 & 96,000 & $29,44 \%$ \\
\hline 3 & 88,000 & $27,00 \%$ \\
\hline 4 & 73,000 & $22,39 \%$ \\
\hline Válidos & 326,000 & $100,00 \%$ \\
\hline
\end{tabular}

Los grupos constituidos corresponden a modelos de profesores con diferente concepción del conocimiento, de la enseñanza y del aprendizaje, con distintos estilos docentes y de evaluación y con diversas habilidades docentes, y sus características son las que se recogen en la Tabla 3.

La diferencia entre el primer modelo y el tercero, ambos constructivistas/centrados en el aprendizaje, está en que en el primero la intensidad de la concepción constructivista es mayor y también en algunas cuestiones relativas a la docencia y a la evaluación: enfoque de la tutoría, uso de las nuevas tecnologías, evaluación inicial, continua y formativa... más presentes en el 1. Este grupo dispone de más habilidades docentes que utiliza en su práctica.

El modelo 3 es un modelo centrado en el aprendizaje, de concepción constructivista, que sin embargo no utiliza con la misma intensidad que el modelo 1 los planteamientos metodológicos coherentes (no se fomenta la autoevaluación en los estudiantes, tampoco se diseña un plan estructurado de tutoría, no se realiza una evaluación inicial, aparece muy poco uso de evaluación continua y formativa, etc.).

La diferencia entre el segundo modelo y el cuarto, ambos más tradicionales/centrados en la enseñanza, está en que en el cuarto se pone un énfasis mayor en la concepción del conocimiento como algo establecido en las disciplinas -no como un proceso de construcción conjunta entre alumnos y profesores- y en su reproducción, así como en la metodología expositiva y en la concepción tradicional de la evaluación mediante el examen. Al igual que en el caso anterior uno de los dos grupos dispone de más habilidades docentes que utiliza en su tarea, y ése es el segundo.

Por otra parte, si prestamos atención a las habilidades docentes de los cuatro modelos, es el modelo 1 el que dispone de más habilidades, seguido por el modelo 2 , éste por el 3 y éste por el 4, que es el que dispone de menos habilidades. 
BERNARDO GARGALLO LÓPEZ, AMPARO FERNÁNDEZ MARCH Y MIGUEL ÁNGEL JIMÉNEZ RODRÍGUEZ

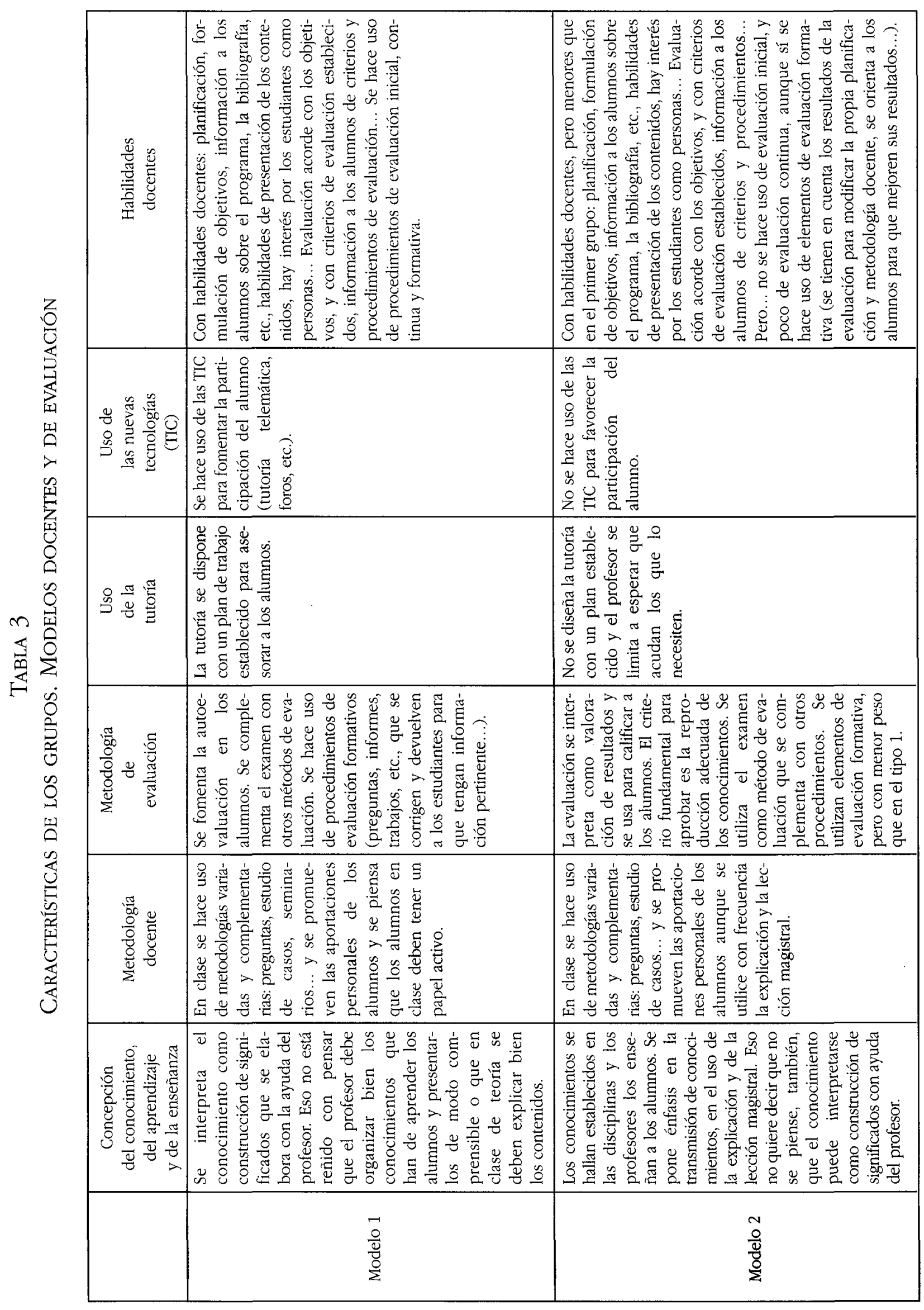




\begin{tabular}{|c|c|c|}
\hline 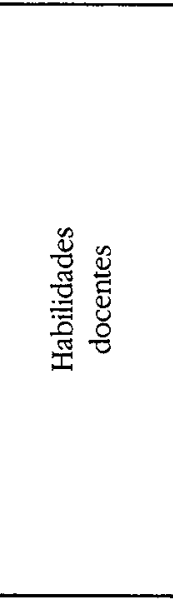 & 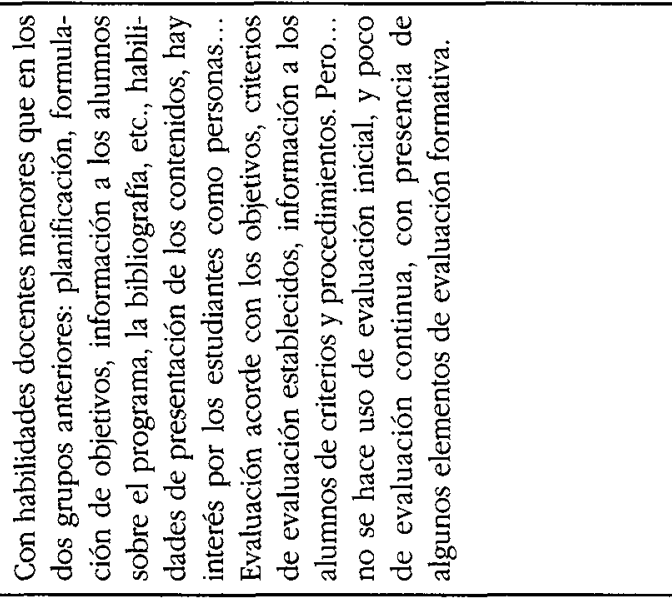 & 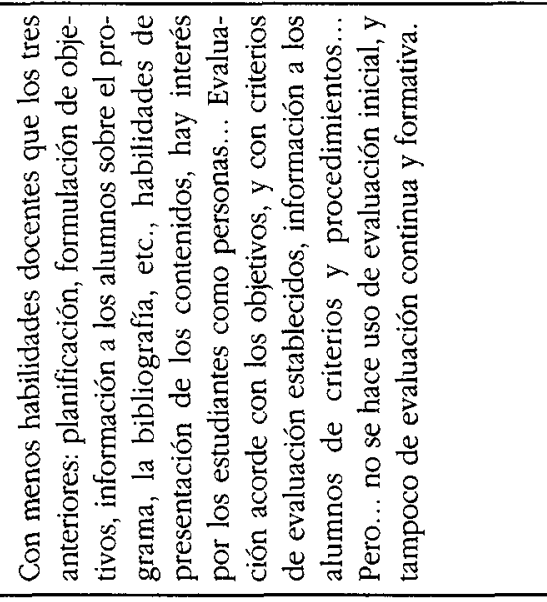 \\
\hline 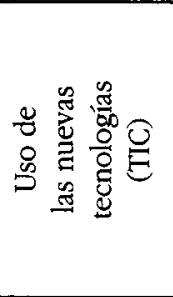 & 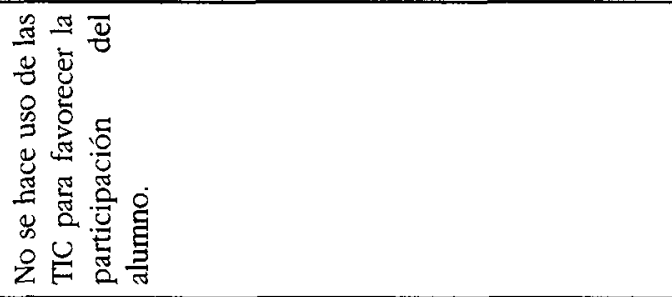 & 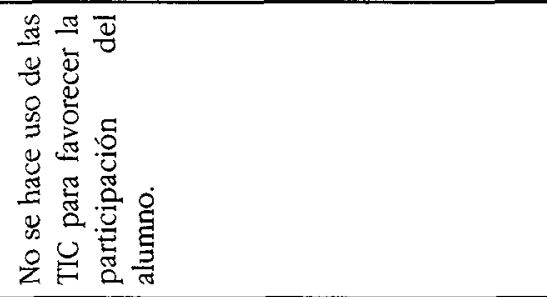 \\
\hline$\frac{8}{3} \frac{\pi}{8}$ & 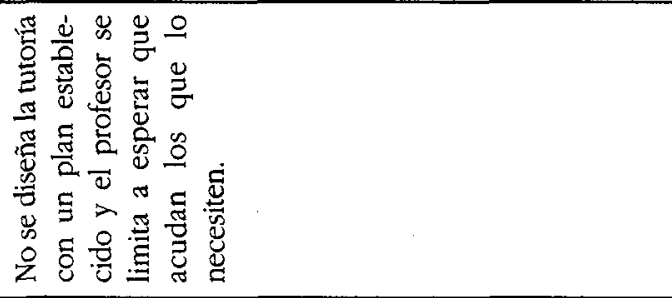 & 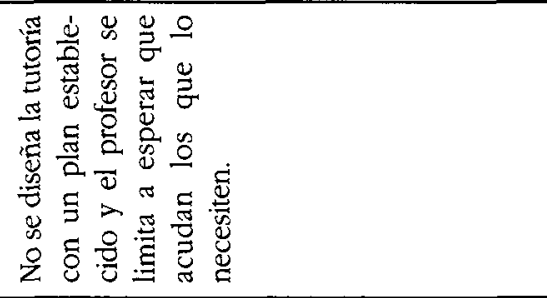 \\
\hline$\frac{\frac{\pi}{60}}{\frac{0}{0}}$ & 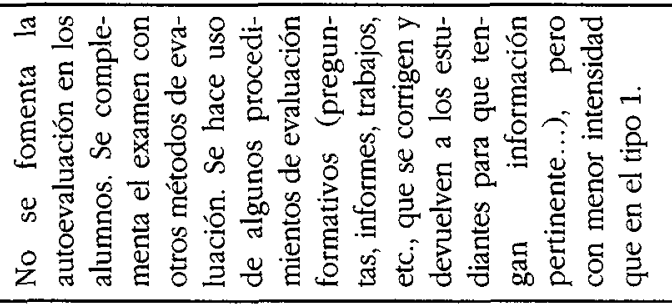 & 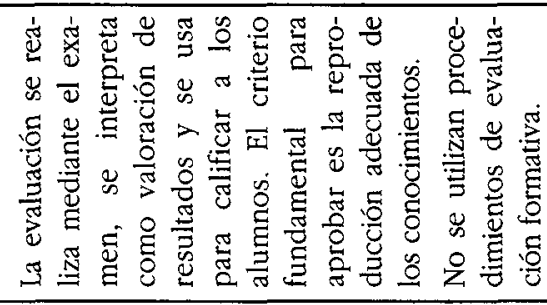 \\
\hline 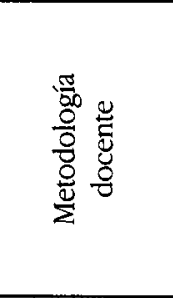 & 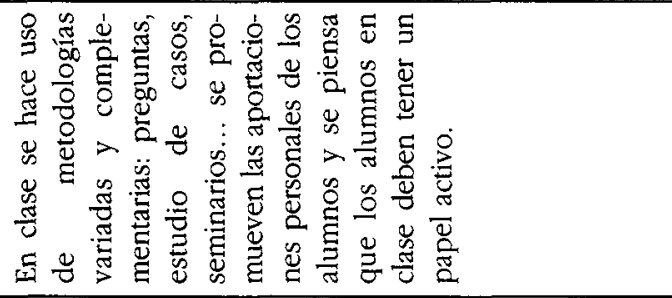 & 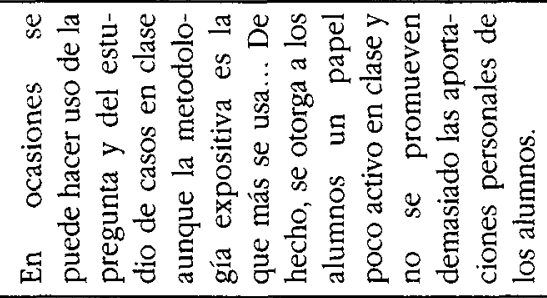 \\
\hline 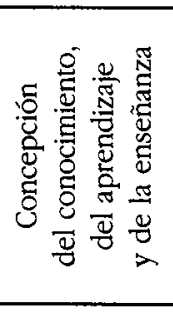 & 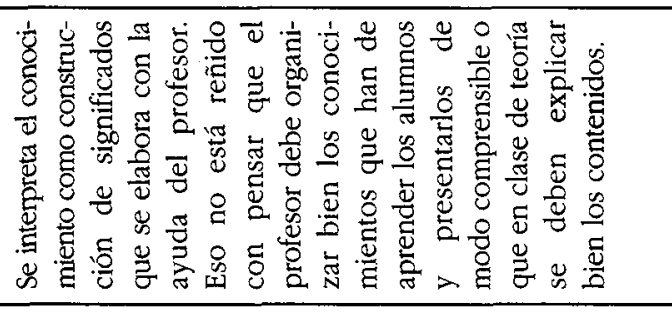 & 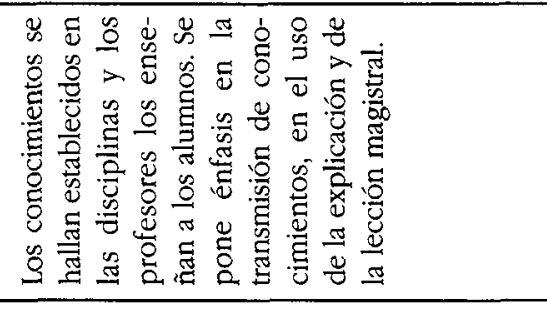 \\
\hline & $\frac{m}{8}$ & 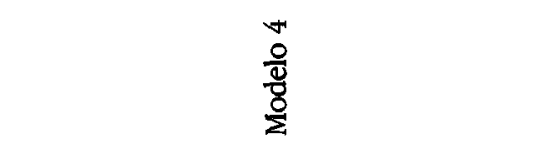 \\
\hline
\end{tabular}


Los datos son coherentes con los hallados por otros investigadores, que constatan dos grandes modelos: el modelo de transmisión del conocimiento o modelo centrado en la enseñanza y el modelo de facilitación del aprendizaje o modelo centrado en el aprendizaje, que en ocasiones presentan variaciones y categorías intermedias (Dall'Alba, 1991; García Valcárcel, 1993; Gow y Kember, 1993; Kember, 1997; Kember y Gow, 1994; Larsson, 1983; Martin y Ramsden, 1992; Samuelowicz y Bain, 1992 y 2001). A diferencia de los últimos datos de Samuelowicz y Bain (2001), nosotros sí encontramos posiciones intermedias.

En nuestro caso, hemos hallado dos modelos, uno centrado en el aprendizaje que actúa de modo coherente con sus concepciones del conocimiento, de la enseñanza y del aprendizaje (el modelo 1) y otro centrado en la enseñanza, tradicional, que actúa también de modo coherente con sus concepciones (el modelo 4).

En el modelo 1 el conocimiento se entiende como construcción conjunta, para la que se cuenta con la ayuda del profesor y de los iguales, se utilizan metodologías de enseñanza variadas y complementarias, se promueve la participación del estudiante al que se da un papel activo, se fomenta la autoevaluación de los alumnos, la evaluación se interpreta como una oportunidad de aprendizaje, se utilizan procedimientos de evaluación formativos, se usa la tutoría de modo planificado, las nuevas tecnologías se utilizan para promover la interacción y el trabajo cooperativo, y se dispone de habilidades docentes que se usan adecuadamente: planificación, información a los estudiantes, buena presentación de los contenidos, hay interés por los estudiantes como personas, se fijan los objetivos y criterios de evaluación, se realiza una evaluación inicial, se utilizan procedimientos de evaluación continua y formativa.

En el modelo 4 se entiende que los conocimientos se hallan establecidos en las disciplinas y los profesores los enseñan a los alumnos. Se pone énfasis en la transmisión de conocimientos, en el uso de la explicación y de la lección magistral. La metodología expositiva es la que más se usa otorgando a los alumnos un papel poco activo en clase. La evaluación se interpreta como valoración de resultados, se usa para calificar a los alumnos y se realiza mediante el examen. Se busca la reproducción de los conocimientos y no se utilizan procedimientos de evaluación formativa. No se diseña un plan para la tutoría, que no es más que un tiempo de permanencia en el despacho. Tampoco se utilizan las TIC para promover la participación de los alumnos. Por último se dispone de menos habilidades docentes: aunque se planifique e informe a los estudiantes, las habilidades para "orquestar" la dinámica del aula son menores y se apoyan básicamente en la lección magistral. No se realiza una evaluación inicial ni tampoco se utilizan procedimientos de evaluación continua y formativa, como ya se ha reflejado antes.

Entre ambos se ubican los otros dos modelos: el modelo 2, centrado en la enseñanza, pero con habilidades docentes y que utiliza planteamientos metodológicos compatibles en ocasiones con el modelo centrado en el aprendizaje, y el modelo 3, centrado en el aprendizaje pero más tibio en su actuación docente, que 
comparte planteamientos similares en este terreno a los que se dan en el modelo tradicional.

Así, en el modelo 2, que es un modelo tradicional, se hace uso de metodologías variadas y complementarias: preguntas, estudio de casos... y se promueven las aportaciones personales de los alumnos, aunque se utilice con frecuencia la explicación y la lección magistral. La evaluación se interpreta como valoración de resultados y se utiliza el examen como método de evaluación pero también se complementa con otros procedimientos. Así mismo, aparecen en el modelo con buena valoración elementos de evaluación formativa, pero con menor peso que en el modelo 1. También es un modelo que dispone de habilidades docentes, aunque menores que en el primer grupo: planificación, formulación de objetivos, información a los alumnos sobre el programa, la bibliografía, etc., habilidades de presentación de los contenidos, hay interés por los estudiantes como personas... La evaluación es acorde con los objetivos, y con criterios de evaluación establecidos, se da información a los alumnos de criterios y procedimientos... Pero... no se hace uso de evaluación inicial, y poco de evaluación continua, aunque sí se hace uso de elementos de evaluación formativa (se tienen en cuenta los resultados de la evaluación para modificar la propia planificación y metodología docente, se orienta a los alumnos para que mejoren sus resultados...).

Por último, en el modelo 3, constructivista y centrado en el aprendizaje, se utilizan procedimientos de enseñanza variados y complementarios aunque menos y con menor intensidad que en el grupo 1. No se fomenta la autoevaluación en los alumnos. Para evaluar se utiliza el examen, que se complementa con otros métodos de evaluación. Se hace uso de procedimientos de evaluación formativos (preguntas, informes, trabajos, etc., que se corrigen y devuelven a los estudiantes para que tengan información pertinente...), pero con menor intensidad que en el tipo 1. No se diseña un plan para la tutoría, que no es más que un tiempo de permanencia en el despacho. Tampoco se utilizan las TIC para promover la participación de los alumnos. Por último se dispone de menos habilidades docentes que los modelos 1 y 2: aunque se planifique e informe a los estudiantes, las habilidades para "orquestar" la dinámica del aula son menores. No se realiza una evaluación inicial y poco los procedimientos de evaluación continua y formativa, como ya se ha reflejado antes.

\section{CONCLUSIONES}

El objetivo fundamental de este trabajo era precisar los modelos de enseñanza y evaluación de los profesores universitarios y sus perfiles en cuanto concepción del conocimiento, de la enseñanza y el aprendizaje, a modalidades de enseñanza, evaluación y a habilidades docentes.

Los resultados obtenidos han permitido el logro del objetivo y son coherentes con los hallados por otros autores, dado que corroboran la existencia de dos 
grandes modelos: un modelo centrado en el aprendizaje y otro centrado en la enseñanza, con dos variantes intermedias. Por otra parte, son un refrendo del constructo teórico que fundamenta el cuestionario elaborado para la validación de la metodología docente y evaluativa de los profesores de universidad. El instrumento (CEMEDEPU) permite evaluar los modelos de docencia y evaluación de los profesores y sus resultados, mediante análisis de conglomerados, dan pie a la constitución de grupos con perfiles coherentes e interpretables.

Por otra parte, los resultados reflejan que hay un porcentaje significativo de profesores no demasiado proclives a planteamientos que sintonicen con un modelo centrado en el aprendizaje, de corte constructivista. De hecho, tales profesores, con una concepción tradicional de la enseñanza y del aprendizaje, más centrada en la enseñanza que en el aprendizaje, con predominio de la lección magistral y de la evaluación sumativa, representan más de la mitad de la muestra (son los pertenecientes a los grupos 2 y 4, un total de 169 profesores, un 51,83\%), aunque es cierto que son los profesores del grupo 4 ( 73 profesores, un 23,39\%) los que detentan un planteamiento más tradicional tanto de concepción como de funcionamiento.

Algo menos de la mitad se sitúa en la otra perspectiva, centrada en el aprendizaje, a nivel de concepción y de funcionamiento con sus alumnos, contando además con buenas habilidades docentes (son los profesores pertenecientes a los grupos 1 y 3, un total de 167 profesores, un 48,17\% de la muestra). Es cierto que es el grupo 3 ( 88 profesores, $27,00 \%$ ) el que tiene una concepción más fuertemente constructivista, con una actuación acorde y con un adecuado repertorio de habilidades docentes.

Ante esta constatación es imprescindible, pues, diseñar una oferta racional de formación para profesores universitarios en formación y también para profesores en ejercicio, adaptada a las necesidades que generan las nuevas políticas de convergencia. No quiere ello decir que los métodos tradicionales de enseñanza no puedan ser eficaces en ciertos casos, siempre que vayan acompañados de las habilidades docentes precisas (planificación, uso de metodologías adecuadas, procedimientos de evaluación rigurosos, etc.), lo que ocurre en el modelo 2 , al que pertenece un grupo importante de profesores, 96, que representan el 29,44\% de la muestra. Se trata de profesionales competentes aunque con una concepción tradicional del aprendizaje y de la enseñanza.

Sin embargo, el problema se plantea especialmente en los casos en que esa concepción tradicional, que entiende la enseñanza como transmisión de conocimientos, centrada en la exposición, etc., no va acompañada de suficientes habilidades docentes que la hagan eficaz, lo que ocurre con el cuarto grupo de profesores. Es el caso de los profesores que se conforman con explicar su disciplina y evaluarla mediante un examen, sin más.

No es aceptable, en ese sentido, que los profesores universitarios puedan ejercer como tales sin pasar por un periodo previo de formación pedagógica. Si bien es cierto que sin saber no se puede enseñar también lo es que no basta con saber 
para saber enseñar. Algunas universidades españolas lo están empezando a entender así y han introducido procesos formativos para profesores noveles, con diversas fórmulas (cursos puntuales, procesos formativos más largos con mentores competentes, etc.), pero no es algo generalizado en todas las universidades ni para todos los profesores en aquellas en las que existen programas de esta índole.

En ese sentido, los proyectos de innovación educativa (P.I.E.s) desarrollados en algunas universidades (la Universidad de Valencia en la que trabajamos es un buen ejemplo) han sido iniciativas realmente estimulantes al propiciar tanto el intercambio de ideas, información y experiencias pedagógicas por parte de los equipos docentes instaurados para su implementación, como el desarrollo de procesos formativos llevados a cabo con el apoyo de la Universidad para su puesta en marcha y funcionamiento. Desde nuestro punto de vista, los procesos de formación continua desarrollados en ese contexto en nuestra Universidad pueden resultar ejemplares, al articularse para dar soluciones a equipos de profesores comprometidos con ganas de avanzar en metodología docente y de evaluación, uso de nuevas tecnologías, aulas virtuales, etc.

En todo caso, las iniciativas tanto de formación inicial como de formación continua para los profesores universitarios han de ser iniciativas sostenidas en el tiempo, estables y con una planificación rigurosa a corto, medio y largo plazo.

Foros privilegiados para el intercambio de experiencias docentes y de innovación en la Universidad son los congresos y reuniones nacionales e internacionales. Sin ir más lejos, el CIDUI (Congreso Internacional de Docencia Universitaria), organizado por las universidades catalanas, que se celebra cada dos años desde el año 2000, en que tuvo lugar la primera edición, ha celebrado este año 2006 su cuarta edición en la Universidad de Barcelona con la asistencia de más de 800 participantes y más de 700 aportaciones. Así mismo son herramientas potentes la constitución de redes, de equipos de investigación, etc.

En definitiva, es un reto que debe abordar la universidad española, que pasa también por prestigiar la docencia -no sólo la investigación-y por darle un peso específico, desde luego superior al que ahora tiene, en la carrera y en la promoción del profesor universitario.

\section{BiBLIOGRAFÍA}

Alonso, F. y MÉnDez, R. M. ${ }^{a}$ (1999) Modelos de enseñanza de los profesores y enfoques de aprendizaje de los estudiantes: Un estudio sobre su relación en la Universidad de Santiago de Compostela, Adaxe, 14-15, 131-147.

Altbach, Ph. G. (2002) Research and training in higher education: the state of the art, Higher Education in Europe, 27 (1-2), 154-168.

Bereiter, C. (1997) Situated cognition and how to overcome it, en KIRSHNER, D. y Whitson, J. A. (eds.). Situated cognition. Social, semiotic and psychological perspectives. Mahwah, NJ, Lawrence Erlbaum, 281-300. 
Dall'Alba, G. (1991) Foreshadowing conceptions of teaching, Studies in Higher Education, 13, 293-297.

ENGELSTRÖM, Y. y COLE, M. (1997) Situated cognition in search of an agenda, en KIRSHNER, D. y Whitson, J. A. (eds.). Situated cognition. Social, semiotic and psychological perspectives. Mahwah, NJ, Lawrence Erlbaum, 301-309.

García ValCárcel, A. (1993) Análisis de los modelos de enseñanza empleados en el ámbito universitario, Revista Española de Pedagogía, 194, 27-53.

Gargalio, B. (2005) En el horizonte del espacio europeo de educación superior. Perfiles de docencia y evaluación de los profesores universitarios. Comunicación presentada en el X Congreso Interuniversitario de Teoría de la Educación. Nuevos espacios y nuevos entornos de educación. Alicante, 11-13 de abril de 2005.

Gow, L. y KemBer, D. (1993) Conceptions of teaching and their relationship to student learning, British Journal of Educational Psychology, 63, 20-33.

KEMBER, D. (1997) A reconceptualisation of the research into university academics' conceptions of teaching, Learning and Instruction, 7, 225-275.

Kember, D. y Gow, L. (1994) Orientations to teaching and their effects on the quality of student learning, Journal of Higher Education, 65 (1), 59-74.

LaRsson, S. (1983) Paradoxes in teaching, Instructional Science, 12 (4), 355-365.

LAVE, J. (1997) The culture of adquisition ant the practice of understanding, en KIRSHNER, D. y WhITSON, J. A. (eds.). Situated cognition. Social, semiotic and psychological perspectives. Mahwah, NJ, Lawrence Erlbaum, 17-35.

LeONTIEv, A. (1978) Actividad, conciencia y personalidad. Buenos Aires, Ciencias del Hombre.

LuRIA, A. R. (1987) Desarrollo bistórico de los procesos cognitivos. Madrid, Akal.

MARTIN, E. y RAMSDEN, P. (1992) An expanding awareness: how lecturers change their understanding of teaching, en PARER, M. S. (ed.). Research and Development in Higher Education, vol. 15. Sidney, HERDSA, 148-155.

Monereo, C. y Pozo, J. I. (2003) La universidad ante la nueva cultura educativa. Madrid, Síntesis. Morris, L.V. (2003) Research Agenda for Higher Education, Innovative Higher Education, 28 (2), 87-90.

Rogoff, B. (1993) Aprendices del pensamiento. El desarrollo cognitivo en el contexto social. Barcelona, Paidós.

Samuelowicz, K. y BaIn, J. D. (1992) Conceptions of teaching held by academic teachers, Higher Education, 22, 229-249.

- (2001) Revisiting academics' beliefs about teaching and learning, Higher Education, 41, 299-325.

- (2002) Identifying academics' orientations to assessment practice, Higher Education, 43, 173-201.

VyGoTsKY, L. S. (1978) Mind in society. The development of higherpsychological process. Cambridge, Ma, Harvard University Press (traducción castellana de S. Furió: El desarrollo de los procesos psicológicos superiores. Barcelona, Crítica, 1979).

Wenger, E. (2001) Comunidades de práctica. Aprendizaje, significado e identidad. Barcelona, Paidós. 